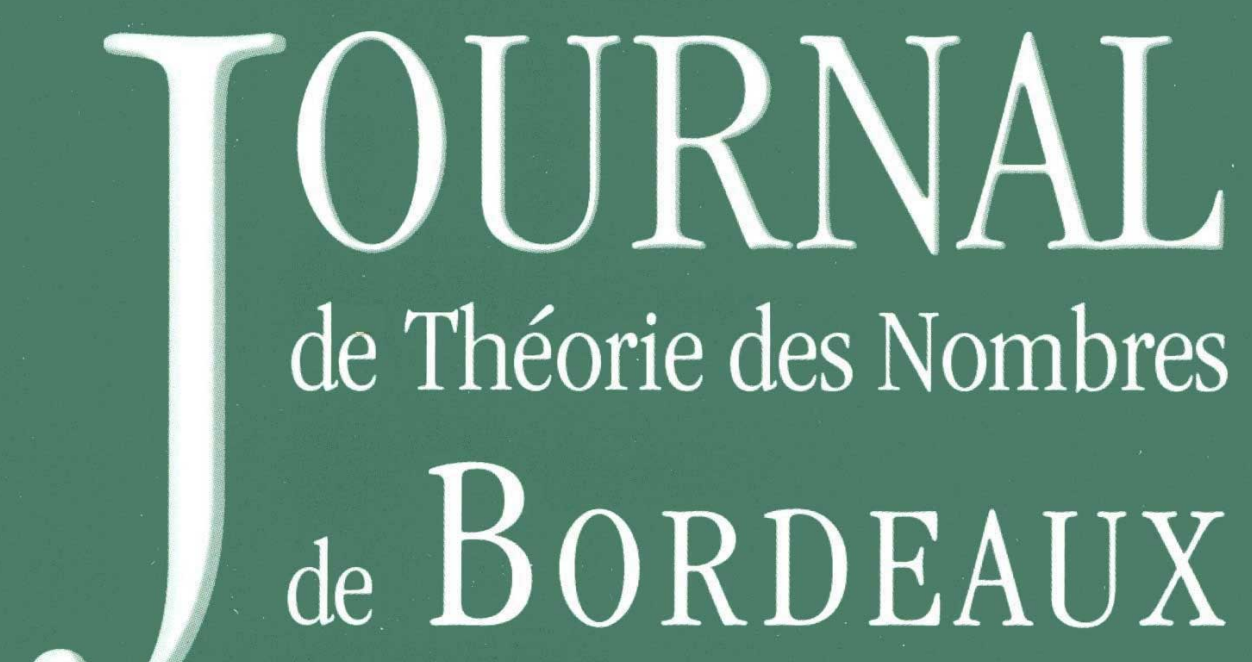

anciennement Séminaire de Théorie des Nombres de Bordeaux

\title{
Yann BUGEAUD
}

\section{Quadratic approximation to automatic continued fractions}

Tome 27, no 2 (2015), p. 463-482.

<http://jtnb.cedram.org/item?id=JTNB_2015__27_2_463_0>

(C) Société Arithmétique de Bordeaux, 2015, tous droits réservés.

L'accès aux articles de la revue «Journal de Théorie des Nombres de Bordeaux » (http://jtnb.cedram.org/), implique l'accord avec les conditions générales d'utilisation (http://jtnb.cedram. org/legal/). Toute reproduction en tout ou partie de cet article sous quelque forme que ce soit pour tout usage autre que l'utilisation à fin strictement personnelle du copiste est constitutive d'une infraction pénale. Toute copie ou impression de ce fichier doit contenir la présente mention de copyright.

\section{cedram}




\title{
Quadratic approximation to automatic continued fractions
}

\author{
par YANN BUGEAUD
}

\begin{abstract}
On the occasion of Axel Thue's 150th birthday
RÉSUMÉ. Nous étudions les ensembles des valeurs prises par les exposants d'approximation quadratique $w_{2}$ et $w_{2}^{*}$ évalués aux nombres réels dont la suite des quotients partiels est engendrée par un automate fini. Entre autres résultats, nous montrons que ces ensembles contiennent tout nombre rationnel suffisamment grand et également des nombres transcendants.
\end{abstract}

ABSTRACT. We study the sets of values taken by the exponents of quadratic approximation $w_{2}$ and $w_{2}^{*}$ evaluated at real numbers whose sequence of partial quotients is generated by a finite automaton. Among other results, we show that these sets contain every sufficiently large rational number and also some transcendental numbers.

\section{Introduction and results}

Throughout this paper, $\mathcal{A}$ denotes a finite or infinite set, called an alphabet, and $\mathcal{A}^{*}$ denotes the set of finite words over $\mathcal{A}$. If $\mathcal{A}$ is finite, a morphism is a map $h$ from $\mathcal{A}^{*}$ into itself such that $h(x y)=h(x) h(y)$ for all finite words $x, y$ in $\mathcal{A}^{*}$. Let $k$ be a positive integer. The morphism $h$ is called $k$-uniform if $h(a)$ has exactly $k$ letters for every $a$ in $\mathcal{A}$. A sequence is $k$-automatic if it is the image, under a coding, of a fixed point of a $k$-uniform morphism. We refer to [10] for equivalent definitions of an automatic sequence and classical results.

Let $b \geq 2$ be an integer. In 1968, Cobham [19] asked whether a real number whose $b$-ary expansion can be generated by a finite automaton (in the sequel, such a real number is called a b-ary automatic number) is always either rational or transcendental. A positive answer to Cobham's question was given in [5], by means of a combinatorial transcendence criterion established in [8]. In [1] we addressed the analogous question for continued fraction expansions and gave a positive answer to it in [18]. Namely, we

Manuscrit reçu le 10 janvier 2014, révisé le 24 juin 2014, accepté le 28 juin 2014.

Mots clefs. Continued fraction, approximation by quadratic numbers, automatic sequence.

Mathematics Subject Classification. 11J70, 11J82. 
proved that a real number whose continued fraction expansion can be generated by a finite automaton (in the sequel, such a real number is called an automatic continued fraction) is either quadratic or transcendental.

It is then natural to study the quality of algebraic approximations to transcendental automatic continued fractions. This quality is measured by means of the functions $w_{d}$ and $w_{d}^{*}$ introduced in 1932 by Mahler [23] and in 1939 by Koksma [21], respectively. Let $d \geq 1$ be an integer and $\xi$ be a real number. We let $w_{d}(\xi)$ denote the supremum of the real numbers $w$ for which

$$
0<|P(\xi)|<H(P)^{-w}
$$

has infinitely many solutions in integer polynomials $P(X)$ of degree at most $d$. Here, $H(P)$ stands for the naïve height of the polynomial $P(X)$, that is, the maximum of the absolute values of its coefficients. We let $w_{d}^{*}(\xi)$ denote the supremum of the real numbers $w^{*}$ for which

$$
0<|\xi-\alpha|<H(\alpha)^{-w^{*}-1}
$$

has infinitely many solutions in algebraic numbers $\alpha$ of degree at most $d$. Here, $H(\alpha)$ stands for the naïve height of the minimal defining polynomial of $\alpha$ over $\mathbf{Z}$. The exponents $w_{1}$ and $w_{1}^{*}$ coincide and, for every real number $\xi$, we have

$$
w_{2}^{*}(\xi) \leq w_{2}(\xi) \leq w_{2}^{*}(\xi)+1
$$

For a proof of (1.1) and more results on $w_{d}$ and $w_{d}^{*}$ the reader is directed to Chapter 3 of [12].

Transcendence measures for non-quadratic automatic continued fractions (more precisely, upper bounds for the functions $w_{d}$ and $w_{d}^{*}$ evaluated at automatic continued fractions) were obtained in [17]. For $d \geq 3$, we applied a general method described in [6,7], while for $d=2$ new arguments were needed, which appeared to have interesting applications to the comparison between Mahler's and Koksma's classifications. In particular, for any $\delta$ in $(0,1]$, we gave [17] explicit examples of real numbers $\xi$ defined by their continued fraction expansion which satisfy $w_{2}(\xi)=w_{2}^{*}(\xi)+\delta$. Furthermore, we established, in a constructive way, that $w_{2}^{*}$ (resp., $w_{2}$ ) takes any value greater than or equal to $(3+\sqrt{17}) / 2$ (resp., $(5+\sqrt{17}) / 2)$. The main purpose of the present work is to discuss Problem 5.4 of [17], recalled below.

Problem 1.1. Determine the set of values taken by the exponents $w_{2}$ and $w_{2}^{*}$ at automatic continued fractions.

Among other results, we give a proof of (a quantitative form of) the assertion following the statement of that problem, claiming that any sufficiently large rational number is a value taken by the functions $w_{2}$ and $w_{2}^{*}$ at automatic continued fractions. 
Theorem 1.2. Let $p / q$ be a rational number greater than $(5+\sqrt{17}) / 2$. Then there exists an automatic continued fraction $\xi_{p / q}$ such that

$$
w_{2}\left(\xi_{p / q}\right)=w_{2}^{*}\left(\xi_{p / q}\right)+1=p / q .
$$

We suspect that every rational number greater than or equal to 2 is a value of $w_{2}$ (resp., $w_{2}^{*}$ ) evaluated at an automatic continued fraction. The analogous result for $b$-ary automatic numbers, established in [13], asserts that, for every integer $b \geq 2$, every rational number greater than or equal to 1 is a value of $w_{1}$ evaluated at a $b$-ary automatic number. The key tool for the proof is the use of continued fractions. Since we do not know any algorithm which would give us all the best quadratic approximations to an automatic continued fraction, it seems to be a difficult problem to replace $(5+\sqrt{17}) / 2$ in the statement of Theorem 1.2 by a smaller value.

We address a related question.

Problem 1.3. To determine the set of values taken by $w_{2}-w_{2}^{*}$ evaluated at automatic continued fractions.

It follows from (1.1) that the set of real numbers defined in Problem 1.3 is contained in the interval $[0,1]$. We are able to prove that this set includes every rational number in $[0,1]$.

Theorem 1.4. For any rational number $\rho$ in $[0,1]$, there exists an automatic continued fraction $\xi_{\rho}$ such that

$$
w_{2}\left(\xi_{\rho}\right)-w_{2}^{*}\left(\xi_{\rho}\right)=\rho .
$$

At present, we do not know a single example of a $b$-ary automatic number at which $w_{1}$ takes an irrational value. It is thus quite tempting to conjecture that the set of values taken by $w_{1}$ at irrational $b$-ary automatic numbers is equal to $[1,+\infty) \cap \mathbf{Q}$. Likewise, we may suspect that the set of values taken by $w_{2}, w_{2}^{*}$ and $w_{2}-w_{2}^{*}$ at automatic numbers does not include irrational numbers. All this would be supported by a result of Schaeffer and Shallit [28] on the Diophantine exponent of automatic sequences, recalled at the end of Section 2.

However, the situation with automatic continued fractions is rather different from that with $b$-ary automatic numbers.

Theorem 1.5. The set of values taken by any of the functions $w_{2}, w_{2}^{*}$ and $w_{2}-w_{2}^{*}$ evaluated at automatic continued fractions includes transcendental numbers.

Theorem 1.5 seems, at first sight, quite surprising. The key observation is that a Lévy constant (see Section 4) does not exist for an arbitrary automatic continued fraction. Furthermore, the values of $w_{2}$ and $w_{2}^{*}$ may depend on the alphabet over which the automatic word is expressed. A 
similar situation does not seem to occur in the case of $b$-ary automatic numbers; see [16] for a discussion.

The proofs of Theorems 1.2, 1.4 and 1.5 are constructive.

We end this section by a further open question.

Problem 1.6. Does there exist an automatic continued fraction $\xi$ for which $w_{2}(\xi)=2$ ?

The present paper is organized as follows. In Section 2 we recall the definitions of the initial critical exponent and the Diophantine exponent of an irrational number, together with their connections to the exponents $w_{2}$ and $w_{2}^{*}$. Some results on continued fractions are given in Section 3. Section 4 is devoted to a discussion of Lévy constants of automatic continued fractions. In Section 5, we recall Liouville's inequality, which bounds the distance between two distinct algebraic numbers from below, and an application. Sections 6 and 7 are devoted to the proofs of our theorem. Quadratic approximation to Thue-Morse continued fractions is discussed in Section 8.

\section{The initial critical exponent and the Diophantine exponent}

The length of a word $W$ over the alphabet $\mathcal{A}$, that is, the number of letters composing $W$, is denoted by $|W|$. For any positive integer $k$, we write $W^{k}$ for the word $W \ldots W$ ( $k$ times repeated concatenation of the word $W)$. More generally, for any positive real number $x$, we let $W^{x}$ denote the word $W^{\lfloor x\rfloor} W^{\prime}$, where $W^{\prime}$ is the prefix of $W$ of length $\lceil(x-\lfloor x\rfloor)|W|\rceil$. Here, and in what follows, $\lfloor y\rfloor$ and $\lceil y\rceil$ denote, respectively, the floor and ceiling of the real number $y$.

Let $\mathbf{a}=\left(a_{\ell}\right)_{\ell \geq 1}$ be a sequence of elements from $\mathcal{A}$ that we identify with the infinite word $a_{1} a_{2} \ldots a_{\ell} \ldots$ The initial critical exponent of a, introduced by Berthé, Holton and Zamboni [11] and denoted by ice(a), is the supremum of the real numbers $x$ for which there exist arbitrarily long prefixes of a that can be expressed in the form $V^{x}$, for a finite word $V$.

The Diophantine exponent of $\mathbf{a}$, introduced in [4] and denoted by Dio(a), is the supremum of the real numbers $\rho$ for which there exist arbitrarily long prefixes of a that can be expressed in the form $U V^{x}$ for some real number $x$ and finite words $U, V$ such that $\left|U V^{x}\right| /|U V| \geq \rho$. It is clear from the definitions that

$$
1 \leq \operatorname{ice}(\mathbf{a}) \leq \operatorname{Dio}(\mathbf{a}) \leq+\infty
$$

and that the initial critical exponent of an ultimately periodic sequence is infinite. However, it is easy to construct sequences whose Diophantine exponent is infinite but which are not ultimately periodic. The Diophantine exponent of a can be viewed as a measure of periodicity of a. We stress that both exponents are independent of the alphabet over which $\mathbf{a}$ is expressed. 
We define the initial critical exponent and the Diophantine exponent of an irrational real number to be, respectively, the initial critical exponent and the Diophantine exponent of its sequence of partial quotients.

Definition 2.1. Let $\xi:=\left[a_{0} ; a_{1}, a_{2}, \ldots, a_{\ell}, \ldots\right]$ be an irrational real number. The initial critical exponent of $\xi$, denoted by ice $(\xi)$, is the initial critical exponent of the infinite word $a_{1} a_{2} \ldots$ The Diophantine exponent of $\xi$, denoted by $\operatorname{Dio}(\xi)$, is the Diophantine exponent of the infinite word $a_{1} a_{2} \ldots$

By truncating the continued fraction expansion of an irrational real number $\xi$ and using the truncation as the period of a periodic continued fraction, one constructs good quadratic approximations to $\xi$ which allow us to bound $w_{2}^{*}(\xi)$ from below. An easy calculation (see Section 11 in [17]) shows that

$$
w_{2}^{*}(\xi) \geq \operatorname{Dio}(\xi)-1,
$$

if $\left(q_{\ell}^{1 / \ell}\right)_{\ell \geq 1}$ converges, where $\left(p_{\ell} / q_{\ell}\right)_{\ell \geq 1}$ denotes the sequence of convergents to $\xi$. If, moreover, $\xi$ has bounded partial quotients, then we also have

$$
w_{2}(\xi) \geq \operatorname{Dio}(\xi) .
$$

and

$$
w_{2}(\xi) \geq w_{2}^{*}(\xi) \geq 2 \text { ice }(\xi)-1
$$

as can be verified by an easy computation.

In all the explicit examples constructed in the present note, we have equality in (2.1) and/or in (2.2). However, we do not have equality in (2.1), (2.2) and (2.3) for all automatic continued fractions $\xi$. Indeed, the Diophantine exponent of an automatic continued fraction can be less than 3 , while, by a result of Davenport and Schmidt [20], we have $w_{2}^{*}(\xi) \geq 2$ for every real number $\xi$ which is not algebraic of degree at most 2 .

We conclude this section with an important result of Schaeffer and Shallit [28], already alluded to in Section 1.

Theorem 2.2. The initial critical exponent and the Diophantine exponent of a transcendental automatic continued fraction are always rational numbers.

Theorem 2.2 speaks in favour of the conjectures discussed in Section 1.

\section{Continued fractions}

We assume that the reader is already familiar with the theory of continued fractions. A classical reference is the monograph of Perron [25]. We just recall two results from [17] and a classical lemma about continuants. Throughout this text, $\bar{a}$ (resp., $\overline{a_{1}, \ldots, a_{n}}$ ) means that the letter $a$ (resp., the $n$ letters $a_{1}, \ldots, a_{n}$ ) is repeated infinitely often.

In this section, the notation $\ll_{a_{1}, \ldots, a_{h}}$ means that the implicit numerical constant only depends on $a_{1}, \ldots, a_{h}$. 
Lemma 3.1. Let $\xi$ be a quadratic real number with ultimately periodic continued fraction expansion

$$
\xi=\left[0 ; a_{1}, \ldots, a_{r}, \overline{a_{r+1}, \ldots, a_{r+s}}\right],
$$

and let $\xi^{\prime}$ denote its Galois conjugate. Then we have

$$
\left|\xi-\xi^{\prime}\right| \geq H(\xi)^{-1} \text {. }
$$

Let $\left(p_{\ell} / q_{\ell}\right)_{\ell \geq 1}$ denote the sequence of convergents to $\xi$. Assume that $r \geq 3$ and $s \geq 1$. If $a_{r-2}, a_{r-1}$ and $a_{r}$ are not greater than $M$ and if $a_{r} \neq a_{r+s}$, then we have

$$
\left|\xi-\xi^{\prime}\right| \ll M^{2} q_{r}^{-2} .
$$

Proof. To establish (3.1), it is sufficient to note that, if the minimal defining polynomial of $\xi$ over $\mathbf{Z}$ is $a X^{2}+b X+c$, then

$$
\left|\xi-\xi^{\prime}\right|=\frac{\sqrt{b^{2}-4 a c}}{a} .
$$

The last assertion of the lemma follows from Lemma 6.1 in [17].

Lemma 3.2. Let $b, c$ and $d$ be distinct positive integers. Let $n \geq 3$ be an integer and $a_{1}, \ldots, a_{n-2}$ be positive integers. Set

$$
\xi:=\left[0 ; a_{1}, \ldots, a_{n-2}, b, c, \bar{b}\right] .
$$

Then the height of $\xi$ satisfies

$$
q_{n}^{2} \ll_{b, c} H(\xi) \ll_{b, c} q_{n}^{2},
$$

where $q_{n}$ is the denominator of the rational number

$$
p_{n} / q_{n}:=\left[0 ; a_{1}, \ldots, a_{n-2}, b, c\right] .
$$

Let $m \geq 3$ be an integer and set

$$
\zeta:=\left[0 ; a_{1}, \ldots, a_{n-2}, b, c, \overline{b, b, \ldots, b, d}\right],
$$

where the periodic part $b, b, \ldots, b, d$ has length $m$. Then the height of $\zeta$ satisfies

$$
q_{n} q_{n+m} \ll_{b, c, d} H(\zeta) \ll_{b, c, d} q_{n} q_{n+m} .
$$

where $q_{n+m}$ is the denominator of $\left[0 ; a_{1}, \ldots, a_{n-2}, b, c, b, b, \ldots, b, d\right]$.

Proof. This is Lemma 6.3 in [17].

If $a_{1}, \ldots, a_{m}$ are positive integers, then, by definition, the continuant $K_{m}\left(a_{1}, \ldots, a_{m}\right)$ is the denominator of the rational number $\left[0 ; a_{1}, \ldots, a_{m}\right]$.

Lemma 3.3. For any positive integers $a_{1}, \ldots, a_{m}$ and any integer $k$ with $1 \leq k \leq m-1$, we have

$$
\begin{gathered}
K_{m}\left(a_{1}, \ldots, a_{m}\right)=K_{m}\left(a_{m}, \ldots, a_{1}\right), \\
K_{m}\left(a_{1}, \ldots, a_{m}\right) \leq\left(1+\max \left\{a_{1}, \ldots, a_{m}\right\}\right)^{m},
\end{gathered}
$$




$$
K_{m}\left(a_{1}, \ldots, a_{m}\right) \geq \max \left\{\left(\min \left\{a_{1}, \ldots, a_{m}\right\}\right)^{m}, 2^{(m-1) / 2}\right\},
$$

and

$$
\begin{aligned}
K_{k}\left(a_{1}, \ldots, a_{k}\right) \cdot K_{m-k}\left(a_{k+1},\right. & \left.\ldots, a_{m}\right) \leq K_{m}\left(a_{1}, \ldots, a_{m}\right) \\
& \leq 2 K_{k}\left(a_{1}, \ldots, a_{k}\right) \cdot K_{m-k}\left(a_{k+1}, \ldots, a_{m}\right) .
\end{aligned}
$$

Proof. See, e.g., on page 15 of [25].

\section{Lévy constants}

Definition 4.1. Let $\xi:=\left[0 ; a_{1}, a_{2}, \ldots\right]$ be an irrational real number and let $\left(p_{\ell} / q_{\ell}\right)_{\ell \geq 1}$ denote the sequence of its convergents. The Lévy constant of $\xi$ is the value

$$
\lim _{\ell \rightarrow+\infty} \frac{\log q_{\ell}}{\ell}
$$

if this limit exists and, otherwise, we say that $\xi$ has no Lévy constant.

Lévy [22] established that $\pi^{2} /(12 \log 2)$ is the Lévy constant of almost all real numbers, in the sense of Lebesgue measure.

We omit the proof of the next lemma, which is a short and easy computation.

Lemma 4.2. For every integer $m \geq 1$ the Lévy constant of the quadratic number $[0 ; \bar{m}]$ is

$$
K_{m}:=\frac{m+\sqrt{m^{2}+4}}{2} .
$$

M. Queffélec [27] proved that a wide class of automatic continued fractions have a Lévy constant. Before stating her result, we need to recall some definitions. A morphism $h$ from $\mathcal{A}^{*}$ into $\mathcal{A}^{*}$ is called primitive if for all letters $a, b$ in $\mathcal{A}$ there exists a positive integer $n$ such that $b$ occurs in $h^{n}(a)$. An automatic sequence is minimal if the underlying (uniform) morphism is primitive. By minimal automatic continued fraction, we mean a real number whose continued fraction expansion can be generated by a primitive uniform morphism. Note that an infinite word $\mathbf{w}$ is minimal if, and only if, corresponding to every block $W$ occurring in $\mathbf{w}$, there exists an integer $k_{W}$ such that every block of length $k_{W}$ of $\mathbf{w}$ contains at least one occurrence of $W$; see Section 10.9 of [10] for additional results.

Theorem 4.3. Every minimal automatic continued fraction has a Lévy constant.

The conclusion of Theorem 4.3 does not hold in general for an automatic continued fraction which is not minimal.

Theorem 4.4. There exist automatic continued fractions which do not have a Lévy constant. 
We point out that there exist transcendental automatic continued fractions which are minimal and have a Lévy constant; see below, in the proof of Theorem 1.2.

To establish Theorem 4.4, we explicitly construct an automatic continued fraction which does not have a Lévy constant. This real number will subsequently be used in the proof of Theorem 1.5.

Proof. Let $a, b, c$ be distinct positive integers. Let $u \geq 1$ and $v \geq 2$ be integers and consider the fixed point $\mathbf{w}$ beginning with $c$ of the $(u+v)$ uniform morphism

$$
a \mapsto a^{u+v}, \quad b \mapsto b^{u+v}, \quad c \mapsto c a^{u} b^{v-1},
$$

namely

$$
\mathbf{w}:=c a^{u} b^{v-1} a^{u(u+v)} \ldots
$$

Let $\xi$ denote the continued fraction associated to $\mathbf{w}$ deprived of its first letter, namely

$$
\xi:=[0 ; a, \ldots, a, b, \ldots, b, a, \ldots],
$$

and let $\left(p_{\ell} / q_{\ell}\right)_{\ell \geq 1}$ be the sequence of its partial quotients. It is easily seen that blocks of the letter $a$ and blocks of the letter $b$ alternate. Precisely, we have

and

$$
w_{(u+v)^{j}}=\cdots=w_{(u+1)(u+v)^{j}-1}=a
$$

$$
w_{(u+1)(u+v)^{j}}=\cdots=w_{(u+v)^{j+1}-1}=b,
$$

for $j \geq 0$.

Let $\varepsilon>0$. It follows from Lemma 3.3 that

$$
\left(K_{a}^{u} K_{b}^{v-1}\right)^{(1-\varepsilon)(u+v)^{j} /(u+v-1)} \leq q_{(u+v)^{j}} \leq\left(K_{a}^{u} K_{b}^{v-1}\right)^{(1+\varepsilon)(u+v)^{j} /(u+v-1)}
$$

and

$$
\begin{aligned}
\left(K_{a}^{u(u+v)} K_{b}^{v-1}\right)^{(1-\varepsilon)(u+v)^{j} /(u+v-1)} & \leq q_{(u+1)(u+v)^{j}} \\
& \leq\left(K_{a}^{u(u+v)} K_{b}^{v-1}\right)^{(1+\varepsilon)(u+v)^{j} /(u+v-1)},
\end{aligned}
$$

when $j$ is large enough. Consequently,

$$
\frac{u}{u+v-1} \log K_{a}+\frac{v-1}{u+v-1} \log K_{b}
$$

and

$$
\frac{u(u+v)}{(u+1)(u+v-1)} \log K_{a}+\frac{v-1}{(u+1)(u+v-1)} \log K_{b}
$$

are limit points of the sequence $\left(\left(\log q_{\ell}\right) / \ell\right)_{\ell \geq 1}$.

Taking, for example, $u=1, v=2$, we get that

$$
\left(K_{a} K_{b}\right)^{1 / 2} \text { and }\left(K_{a}^{3} K_{b}\right)^{1 / 4}
$$


are distinct limit points of $\left(q_{\ell}^{1 / \ell}\right)_{\ell \geq 1}$. This provides us with a family of explicit examples of automatic continued fractions which do not have a Lévy constant.

\section{Liouville's inequality and an application}

By Liouville's inequality, we mean a non-trivial lower bound for the distance between two distinct algebraic numbers. For instance, it follows from Theorem A.1 of [12] that

$$
|\alpha-\beta| \geq 10^{-2} \cdot H(\alpha)^{-2} \cdot H(\beta)^{-2}
$$

holds for distinct algebraic numbers $\alpha$ and $\beta$ of degree at most two. When the Galois conjugate of $\alpha$ is very close to $\alpha$, then (5.1) can be considerably improved; see [17].

Furthermore, it is well known that if a real number $\xi$ has a dense (in a suitable sense) sequence of very good algebraic approximations, then $w_{2}^{*}(\xi)$ can be determined. A precise statement, which relies on a refinement of (5.1), is Lemma 7.3 from [17], recalled below. It also includes results on $w_{2}(\xi)$.

Lemma 5.1. Let $\xi$ be a real number. Assume that there exist positive real numbers $c_{1}, c_{2}, c_{3}, \delta, \rho, \theta$ and a sequence $\left(\alpha_{j}\right)_{j \geq 1}$ of quadratic numbers such that

$$
c_{1} H\left(\alpha_{j}\right)^{-\rho-1} \leq\left|\xi-\alpha_{j}\right| \leq c_{2} H\left(\alpha_{j}\right)^{-\delta-1}
$$

and

$$
H\left(\alpha_{j}\right) \leq H\left(\alpha_{j+1}\right) \leq c_{3} H\left(\alpha_{j}\right)^{\theta}
$$

for $j \geq 1$. Set $\varepsilon=0$ or assume that there exist $c_{4} \geq 1$ and $0<\varepsilon \leq 1$ such that

$$
\left|\alpha_{j}-\alpha_{j}^{\prime}\right| \leq c_{4} H\left(\alpha_{j}\right)^{-\varepsilon}
$$

for $j \geq 1$, where $\alpha_{j}^{\prime}$ denotes the Galois conjugate of $\alpha_{j}$. Then we have

$$
\delta \leq w_{2}^{*}(\xi) \leq \rho
$$

when

$$
(\rho-1)(\delta-1+\varepsilon) \geq 2 \theta(2-\varepsilon) .
$$

Furthermore, if $\varepsilon>0$, then we have

$$
\delta \leq w_{2}^{*}(\xi) \leq \rho \quad \text { and } \quad w_{2}(\xi)=w_{2}^{*}(\xi)+\varepsilon,
$$

when

$$
(\delta-2+\varepsilon)(\delta-1+\varepsilon) \geq 2 \theta(2-\varepsilon)
$$

and

$$
\lim _{j \rightarrow+\infty} \frac{\log \left|\alpha_{j}-\alpha_{j}^{\prime}\right|}{\log H\left(\alpha_{j}\right)}=-\varepsilon
$$




\section{Proofs of Theorems 1.2 and 1.4}

Proofs of Theorem 1.2 and the case $\rho=1$ of Theorem 1.4.

Let $p / q$ be a rational number greater than $(5+\sqrt{17}) / 2$ and let $I$ be a closed interval in $((5+\sqrt{17}) / 2, p / q)$, whose center $\theta$ is irrational. Let $b$ be an integer such that $\theta+p^{-b}$ is in $I$. Let $a \geq 3$ and $J \geq 3$ be integers such that

$$
\theta<q p^{a-1}\left(\theta+p^{-b}\right)^{-J}<\theta+p^{-b} .
$$

These numbers exist since the sequence $\left(p^{a} /\left(\theta+p^{-b}\right)^{J}\right)_{a, J \geq 3}$ is dense in the set of positive real numbers.

Set $r_{0}:=p^{b}$ and $r_{1}:=\left\lceil\theta p^{b}\right\rceil$. For $j=2, \ldots, J$, set $r_{j}=\left\lceil\theta r_{j-1}\right\rceil$.

Observe that

$$
r_{j}-1<\theta r_{j-1}<r_{j}, \quad j=1, \ldots, J
$$

hence,

$$
\theta<\frac{r_{j}}{r_{j-1}}<\theta+\frac{1}{r_{j-1}} \leq \theta+p^{-b}, \quad j=1, \ldots, J .
$$

Consequently,

$$
\frac{r_{1}}{p^{b}}, \frac{r_{2}}{r_{1}}, \ldots, \frac{r_{J}}{r_{J-1}}
$$

are all in $I$. Since

$$
\frac{q p^{a+b-1}}{r_{J}}=q p^{a-1} \times \frac{r_{J-1}}{r_{J}} \times \cdots \times \frac{r_{1}}{r_{2}} \times \frac{p^{b}}{r_{1}},
$$

we deduce from (6.1) and (6.2) that

$$
\theta<q p^{a-1}\left(\theta+p^{-b}\right)^{-J} \leq \frac{q p^{a+b-1}}{r_{J}} \leq q p^{a-1} \theta^{-J}<\theta+p^{-b} ;
$$

thus the rational number $q p^{a+b-1} / r_{J}$ belongs to the interval $I$.

Define the sequence $\mathbf{y}=\left(y_{n}\right)_{n \geq 1}$ by setting $y_{n}=2$ if $n$ is of the form $p^{h a} r_{j}$ or $q p^{(h+1) a+b-1}$ for some integers $h \geq 0$ and $j=0, \ldots, J$, and setting $y_{n}=1$ otherwise. It follows from Theorem 5.6.3 of [10] that $\mathbf{y}$ is an automatic sequence. The first indices $n$ at which $y_{n}=2$ are

$$
p^{b}, r_{1}, r_{2}, \ldots, r_{J}, q p^{a+b-1}, p^{a+b}, p^{a} r_{1}, p^{a} r_{2}, \ldots, p^{a} r_{J}, q p^{2 a+b-1}, p^{2 a+b}, \ldots
$$

The quotient of any two consecutive elements of the latter sequence is always greater than $(5+\sqrt{17}) / 2$ and at most equal to $p / q$, with equality for infinitely many indices. The Diophantine exponent of $\mathbf{y}$ is then equal to $p / q$. Define

$$
\xi_{p / q}:=\left[0 ; y_{1}, y_{2}, \ldots, y_{n}, \ldots\right]=[0 ; 1,1, \ldots, 1,2,1, \ldots] .
$$

Let $\left(n_{k}\right)_{k \geq 1}$ denote the increasing sequence of integers $n$ such that $y_{n}=2$. For $k \geq 1$, set

$$
\xi_{k}:=\left[0 ; y_{1}, \ldots, y_{n_{k}}, \overline{1}\right]
$$


By Lemmas 3.2 and 3.3 and classical results on continued fractions, we have

$$
q_{n_{k+1}} \asymp q_{n_{k}} K_{1}^{n_{k+1}-n_{k}}, \quad H\left(\xi_{k}\right) \asymp q_{n_{k}}^{2} \quad \text { and } \quad\left|\xi_{p / q}-\xi_{k}\right| \asymp q_{n_{k+1}}^{-2} .
$$

Consequently, there exists an absolute constant $c_{1}$ such that $H\left(\xi_{k+1}\right) \leq$ $c_{1} H\left(\xi_{k}\right)^{p / q}$. Define $\varphi_{k}$ by $H\left(\xi_{k+1}\right)=c_{1} H\left(\xi_{k}\right)^{\varphi_{k}}$. Observe that $\varphi_{k} \leq p / q$ and that $\varphi_{k}>(5+\sqrt{17}) / 2$ when $k$ is sufficiently large.

Let $\zeta$ be a quadratic real number with large height and let $k$ be the integer defined by $H\left(\xi_{k}\right) \leq H(\zeta)<H\left(\xi_{k+1}\right)$. The constants $c_{2}, c_{3}, \ldots$ below are absolute. By (5.1) and the triangle inequality, we have

$$
\left|\xi_{p / q}-\zeta\right| \geq 10^{-2} \cdot H\left(\xi_{k}\right)^{-2} \cdot H(\zeta)^{-2}-\left|\xi_{p / q}-\xi_{k}\right|
$$

and we deduce that $\left|\xi_{p / q}-\zeta\right| \geq c_{2} H(\zeta)^{-4}$ as soon as

$$
H(\zeta) \leq c_{3} H\left(\xi_{k}\right)^{\varphi_{k}-1}
$$

Likewise, using the inequality

$$
\left|\xi_{p / q}-\zeta\right| \geq 10^{-2} \cdot H\left(\xi_{k+1}\right)^{-2} \cdot H(\zeta)^{-2}-\left|\xi_{p / q}-\xi_{k+1}\right|,
$$

we see that $\left|\xi_{p / q}-\zeta\right| \geq c_{4} H(\zeta)^{-p / q}$ whenever

$$
H(\zeta) \geq c_{5} H\left(\xi_{k}\right)^{2 \varphi_{k} /(-2+p / q)} .
$$

Since $p / q \geq \varphi_{k}>(5+\sqrt{17}) / 2$ (here, we use that $H(\xi)$, hence $k$, is sufficiently large), we get

$$
2 \varphi_{k}<(-2+p / q)\left(\varphi_{k}-1\right) .
$$

By (6.4) and (6.5), this shows that every quadratic number $\zeta$ with sufficiently large height and which does not belong to the sequence $\left(\xi_{k}\right)_{k \geq 1}$ satisfies

$$
\left|\xi_{p / q}-\zeta\right| \geq c_{6} H(\zeta)^{-p / q}
$$

Furthermore, there are arbitrarily large integers $k$ for which $n_{k+1} / n_{k}=p / q$ and

$$
\left|\xi_{p / q}-\xi_{k}\right| \leq c_{7} H\left(\xi_{k}\right)^{-p / q}
$$

We conclude that $w_{2}^{*}\left(\xi_{p / q}\right)=p / q-1$. It follows from Lemma 3.1 that $\left|\xi_{k}-\xi_{k}^{\prime}\right| \leq c_{8} H\left(\xi_{k}\right)^{-1}$ for $k \geq 1$. Thus, our construction shows that every very good quadratic approximation to $\xi_{p / q}$ has its Galois conjugate also very close to $\xi_{p / q}$, hence, we deduce that $w_{2}\left(\xi_{p / q}\right)=p / q$, as claimed. Also note that the automatic continued fraction $\xi$ is not minimal, while (6.3) implies that $\log K_{1}$ is the Lévy constant of $\xi$.

Proof of the case $\rho=0$ of Theorem 1.4.

Let $v \geq 2$ be an integer and let $\mathbf{a}_{v}=a_{1, v} a_{2, v} \ldots$ denote the fixed point of the $(2 v)$-uniform morphism

$$
2 \mapsto(23)^{v}, \quad 3 \mapsto(23)^{v-1} 24, \quad 4 \mapsto 2^{2 v} .
$$


For $k \geq 0$, let $U_{k}$ denote the prefix of $\mathbf{a}_{v}$ of length $2 \cdot(2 v)^{k}$. Observe that $U_{k}^{2 v-1 / 2}$ is a prefix of $\mathbf{a}_{v}$.

Set $\xi_{k}:=\left[0 ; U_{k}, U_{k}, \ldots\right]$. Observe that

$$
H\left(\xi_{k}\right) \leq 5^{\left|U_{k}\right|}
$$

and

$$
\left|\xi-\xi_{k}\right| \leq 2^{-(4 v-1)\left|U_{k}\right|} \leq 2^{-3 v\left|U_{k}\right|} \leq 5^{-c v\left|U_{k}\right|} \leq H\left(\xi_{k}\right)^{-c v},
$$

with $c=(3 \log 2) /(\log 5)$. Note that, here and below, we make no effort to refine the estimates.

Let $\zeta$ be a quadratic number of sufficiently large height and with $\zeta \neq \xi_{k}$ for every $k \geq 1$. Let $k$ be such that

$$
5^{\left|U_{k}\right|} \leq H(\zeta)<5^{\left|U_{k+1}\right|}=5^{2 v\left|U_{k}\right|} .
$$

We deduce from (5.1) that

$$
|\xi-\zeta| \geq\left|\zeta-\xi_{k}\right|-\left|\xi-\xi_{k}\right| \geq 10^{-2} \cdot H(\zeta)^{-2} 5^{-2\left|U_{k}\right|}-5^{-c v\left|U_{k}\right|}
$$

thus,

$$
|\xi-\zeta| \geq 10^{-3} \cdot H(\zeta)^{-4}
$$

as soon as

$$
H(\zeta) \leq 10^{-3} \cdot 5^{(c v-2)\left|U_{k}\right| / 2} .
$$

On the other hand, assuming that $v>12 c$ (which ensures that $c v \geq 3+$ $(8 v /(c v-2)))$, we get again by $(5.1)$ that

$$
\begin{aligned}
|\xi-\zeta| & \geq\left|\zeta-\xi_{k+1}\right|-\left|\xi-\xi_{k+1}\right| \\
& \geq 10^{-2} \cdot H(\zeta)^{-2} 5^{-4 v\left|U_{k}\right|}-5^{-2 c v^{2}\left|U_{k}\right|} \geq 10^{-3} \cdot H(\zeta)^{-2} 5^{-4 v\left|U_{k}\right|},
\end{aligned}
$$

and we deduce that

$$
|\xi-\zeta| \geq\left(10^{3} H(\zeta)\right)^{-2-8 v /(c v-2)}
$$

when (6.6) does not hold.

Since $c v \geq 2+(8 v /(c v-2))$, the sequence $\left(\xi_{k}\right)_{k \geq 1}$ comprises all the best approximants to $\xi$ and we get

$$
w_{2}^{*}(\xi)=\limsup _{k \rightarrow+\infty} \frac{-\log \left|\xi-\xi_{k}\right|}{\log H\left(\xi_{k}\right)}-1 .
$$

We do not know the exact value of $w_{2}^{*}(\xi)$. We only know that, since the Galois conjugate $\xi_{k}^{\prime}$ of $\xi_{k}$ is negative, it satisfies $\left|\xi_{k}-\xi_{k}^{\prime}\right| \geq 1 / 3$. Combined with the fact that the other approximants to $\xi$ are much less good (since $c v \geq 3+(8 v /(c v-2)))$, it follows from $(1.1)$ that $w_{2}(\xi)=w_{2}^{*}(\xi)$.

Completion of the proof of Theorem 1.4.

To complete the proof of Theorem 1.4, it then remains for us to treat the case where $\rho$ is in $(0,1) \cap \mathbf{Q}$. 
Let $r / s$ be a rational number in $(0,1)$ with $r$ and $s$ positive, and set

$$
\eta=\frac{2(s-r)}{r} \text {. }
$$

Let $a$ be a positive integer. We consider the sequence

$$
r^{a}, r^{a}(1+\eta), \ldots, r^{a}(1+m \eta), r^{2 a}, r^{2 a}(1+\eta), \ldots, r^{2 a}(1+m \eta), r^{3 a}, \ldots
$$

where $m$ is the largest integer such that $1+m \eta<r^{a}$, that is, the largest integer smaller than $r\left(r^{a}-1\right) /(2(s-r))$.

Let $\left(y_{n}\right)_{n \geq 1}$ be the sequence defined by setting:

$y_{n}=2$, if there is an integer $k \geq 1$ such that $n=r^{a k}$;

$y_{n}=3$, if there are integers $j=1, \ldots, m$ and $k \geq 1$ such that $n=r^{a k}(1+$ $j \eta)$

$y_{n}=1$, otherwise.

It follows from Theorem 5.6.3 and Corollary 5.4.5 of [10] that $\left(y_{n}\right)_{n \geq 1}$ is an automatic sequence. Set

$$
\xi:=\left[0 ; y_{1}, y_{2}, \ldots, y_{n}, \ldots\right] .
$$

Let $\left(p_{\ell} / q_{\ell}\right)_{\ell \geq 1}$ denote the sequence of convergents to $\xi$. For $k \geq 1$,

$$
\xi_{k}:=\left[0 ; y_{1}, y_{2}, \ldots, y_{r^{a k}-1}, 2, \overline{1, \ldots, 1,3}\right]
$$

is a good approximant to $\xi$. Let $\varepsilon$ be a real number in $(0,0.1)$. By Lemmas 3.2 and 3.3 and the theory of continued fractions,

$$
K_{1}^{-2 r^{a(k+1)}(1+\varepsilon)} \leq\left|\xi-\xi_{k}\right| \leq K_{1}^{-2 r^{a(k+1)}(1-\varepsilon)}
$$

and

$$
K_{1}^{(2+\eta) r^{a k}(1-\varepsilon)} \leq H\left(\xi_{k}\right) \leq K_{1}^{(2+\eta) r^{a k}(1+\varepsilon)}
$$

hold when $k$ is sufficiently large. Furthermore, it follows from Lemma 3.1 that the Galois conjugate $\xi_{k}^{\prime}$ of $\xi_{k}$ satisfies

$$
K_{1}^{-2 r^{a k}(1+\varepsilon)} \leq\left|\xi_{k}-\xi_{k}^{\prime}\right| \leq K_{1}^{-2 r^{a k}(1-\varepsilon)},
$$

provided that $k$ is large enough. We deduce from Lemma 5.1 applied with $\theta=r^{a}(1+3 \varepsilon)$ that

$$
w_{2}^{*}(\xi)=\frac{2 r^{a}}{2+\eta}-1 \quad \text { and } \quad w_{2}(\xi)=\frac{2 r^{a}-\eta}{2+\eta},
$$

when $a$ is sufficiently large; thus,

$$
w_{2}(\xi)-w_{2}^{*}(\xi)=\frac{2}{2+\eta}=\frac{r}{s} .
$$

The proof of Theorem 1.4 is complete. 


\section{Proof of Theorem 1.5}

To show that the exponents $w_{2}$ and $w_{2}^{*}$ take transcendental values at some automatic continued fractions, we consider the family of automatic continued fractions defined in the proof of Theorem 4.4. We keep the same notation. For $j \geq 1$, set

$$
\alpha_{j}:=\left[0 ; w_{1}, \ldots, w_{(u+v)^{j}-1}, \bar{a}\right]
$$

and

$$
\beta_{j}:=\left[0 ; w_{1}, \ldots, w_{(u+1)(u+v)^{j}-1}, \bar{b}\right] .
$$

It follows from Lemma 3.2 that

$$
H\left(\alpha_{j}\right) \asymp q_{(u+v)^{j}}^{2} \quad \text { and } \quad H\left(\beta_{j}\right) \asymp q_{(u+1)(u+v)^{j}}^{2},
$$

where (as below) the numerical constants implied by $\asymp$ depend only on $a$ and $b$. Observe that

$$
\left|\xi-\alpha_{j}\right| \asymp q_{(u+1)(u+v)^{j}}^{-2} \asymp H\left(\beta_{j}\right)^{-1}
$$

and

$$
\left|\xi-\beta_{j}\right| \asymp q_{(u+v)^{j+1}}^{-2} \asymp H\left(\alpha_{j+1}\right)^{-1} .
$$

We need to precisely estimate the growth of the sequence $\left(q_{\ell}\right)_{\ell \geq 1}$. It follows from the calculation performed in the proof of Theorem 4.4 that

$$
\alpha:=\limsup _{j \rightarrow+\infty} \frac{-\log \left|\xi-\alpha_{j}\right|}{\log H\left(\alpha_{j}\right)}=\frac{\log \left(K_{a}^{u(u+v)} K_{b}^{v-1}\right)}{\log \left(K_{a}^{u} K_{b}^{v-1}\right)}
$$

and

$$
\beta:=\limsup _{j \rightarrow+\infty} \frac{-\log \left|\xi-\beta_{j}\right|}{\log H\left(\beta_{j}\right)}=\frac{\log \left(K_{a}^{u(u+v)} K_{b}^{(u+v)(v-1)}\right)}{\log \left(K_{a}^{u(u+v)} K_{b}^{v-1}\right)} .
$$

We select $u, v, a$ and $b$ in such a way that $\alpha$ and $\beta$ are both large. To do this, observe first that $\alpha \beta=u+v$, choose $u$ large and set $v=u+1$. This gives

$$
\alpha=\frac{\log \left(K_{a}^{2 u+1} K_{b}\right)}{\log \left(K_{a} K_{b}\right)} \quad \text { and } \quad \beta=\frac{\log \left(K_{a}^{2 u+1} K_{b}^{2 u+1}\right)}{\log \left(K_{a}^{2 u+1} K_{b}\right)} .
$$

Now choose $b$ equal to $\left\lfloor a^{\sqrt{u}}\right\rfloor$ or $\left\lfloor a^{\sqrt{u}}\right\rfloor+1$, in such a way that $\sqrt{a}$ and $\sqrt{b}$ do not belong to the same quadratic field. Observe that $\alpha$ and $\beta$ are then of the order of magnitude of $\sqrt{u}$. Furthermore,

$$
\log H\left(\alpha_{j}\right) \asymp \frac{2(u+v)^{j}}{u+v-1}\left(u \log K_{a}+(v-1) \log K_{b}\right)
$$

is of the order of magnitude of $u^{j} \sqrt{u}$ and

$$
\log H\left(\beta_{j}\right) \asymp \frac{2(u+v)^{j}}{u+v-1}\left(u(u+v) \log K_{a}+(v-1) \log K_{b}\right)
$$


is of the order of magnitude of $u^{j+1}$. The quotients

$$
\frac{\log H\left(\beta_{j}\right)}{\log H\left(\alpha_{j}\right)} \quad \text { and } \quad \frac{\log H\left(\alpha_{j+1}\right)}{\log H\left(\beta_{j}\right)}
$$

are both of the order of magnitude of $\sqrt{u}$. Lemma 5.1 then shows that, provided that $u$ is sufficiently large, all the best approximations to $\xi$ belong to the sequences $\left(\alpha_{j}\right)_{j \geq 1}$ and $\left(\beta_{j}\right)_{j \geq 1}$. Keeping in mind that, by (7.1) and Lemma 3.1, the $\alpha_{j}$ 's and the $\beta_{j}$ 's are very close to their Galois conjugate, we get

$$
w_{2}^{*}(\xi)+1=w_{2}(\xi)=\max \{\alpha, \beta\} .
$$

Note that $\alpha$ and $\beta$ are quotients of logarithms of real algebraic numbers greater than 1 . Thus, by the Gelfond-Schneider theorem, they are rational or transcendental. Here, they are transcendental since $\sqrt{a}$ and $\sqrt{b}$ do not belong to the same quadratic field.

Similar ideas can be used to prove that the function $w_{2}-w_{2}^{*}$ can take transcendental values at automatic continued fractions.

Let $a, b, c, d$ be distinct positive integers. Let $s, t, u$ be integers greater than or equal to 2 and consider the fixed point $\mathbf{w}$ beginning with $d$ of the $(t+s u)$-uniform morphism

$$
a \mapsto a^{t+s u}, \quad b \mapsto b^{t+s u}, \quad c \mapsto b^{t+s u-1} c, \quad d \mapsto d a^{t-1}\left(b^{u-1} c\right)^{s},
$$

namely,

$$
\mathbf{w}:=d a^{t-1} b^{u-1} c b^{u-1} c \ldots
$$

We assume that $t$ is large compared to $s u$.

Denote by $\xi$ the continued fraction associated with $\mathbf{w}$ deprived of its first letter, namely

$$
\xi:=[0 ; a, \ldots, a, b, \ldots, b, c, b, \ldots],
$$

and let $\left(p_{\ell} / q_{\ell}\right)_{\ell \geq 1}$ be its sequence of partial quotients. For $j \geq 0$ and $h=$ $0,1, \ldots, s-1$, we have

$$
\begin{gathered}
w_{(t+s u)^{j}}=\cdots=w_{t(t+s u)^{j}-1}=a, \\
w_{(t+h u)(t+s u)^{j}}=\cdots=w_{(t+(h+1) u)(t+s u)^{j}-2}=b,
\end{gathered}
$$

and

$$
w_{(t+(h+1) u)(t+s u)^{j-1}}=c .
$$

For $j \geq 1$, set

$$
\gamma_{j}:=\left[0 ; w_{1}, \ldots, w_{t(t+s u)^{j}-1}, \overline{b, \ldots, b, c}\right]
$$


Let $\varepsilon$ be a positive real number. Lemma 3.2 and a rapid calculation show that

$$
\begin{aligned}
& \left(K_{a}^{2(t-1)(t+s u)} K_{b}^{2 s u+u}\right)^{(1-\varepsilon)(t+s u)^{j} /(t+s u-1)} \leq H\left(\gamma_{j}\right) \\
& \quad \leq\left(K_{a}^{2(t-1)(t+s u)} K_{b}^{2 s u+u}\right)^{(1+\varepsilon)(t+s u)^{j} /(t+s u-1)}
\end{aligned}
$$

and

$$
\begin{aligned}
\left(K_{a}^{2(t-1)} K_{b}^{2 s u}\right)^{(1-\varepsilon)(t+s u)^{j+1} /(t+s u-1)} & \leq-\log \left|\xi-\gamma_{j}\right| \\
\leq & \left(K_{a}^{2(t-1)} K_{b}^{2 s u}\right)^{(1+\varepsilon)(t+s u)^{j+1} /(t+s u-1)}
\end{aligned}
$$

when $j$ is large enough. Consequently,

$\lim _{j \rightarrow+\infty} \frac{-\log \left|\xi-\gamma_{j}\right|}{\log H\left(\gamma_{j}\right)}-1=\frac{2(t-1)(t+s u) \log K_{a}+2 s u(t+s u) \log K_{b}}{2(t-1)(t+s u) \log K_{a}+(2 s u+u) \log K_{b}}-1$

$$
=\frac{(2 s u(t+s u-1)-u) \log K_{b}}{2(t-1)(t+s u) \log K_{a}+(2 s u+u) \log K_{b}} .
$$

Furthermore, the Galois conjugate $\gamma_{j}^{\prime}$ of $\gamma_{j}$ satisfies

$$
\begin{aligned}
& \left(K_{a}^{2(t-1)(t+s u)} K_{b}^{2 s u}\right)^{(1-\varepsilon)(t+s u)^{j} /(t+s u-1)} \leq-\log \left|\xi-\gamma_{j}^{\prime}\right| \\
& \leq\left(K_{a}^{2(t-1)(t+s u)} K_{b}^{2 s u}\right)^{(1+\varepsilon)(t+s u)^{j} /(t+s u-1)},
\end{aligned}
$$

again when $j$ is large enough. Consequently, letting $P_{j}(X)$ denote the minimal defining polynomial of $\gamma_{j}$ over $\mathbf{Z}$, we get

$$
\lim _{j \rightarrow+\infty} \frac{-\log \left|P\left(\gamma_{j}\right)\right|}{\log H\left(\gamma_{j}\right)}=\frac{2(t-1)(t+s u) \log K_{a}+(2 s u(t+s u)-u) \log K_{b}}{2(t-1)(t+s u) \log K_{a}+(2 s u+u) \log K_{b}} .
$$

Observe also that

$$
\lim _{j \rightarrow+\infty} \frac{\log H\left(\gamma_{j+1}\right)}{\log H\left(\gamma_{j}\right)}=t+s u
$$

Choosing our parameters in such a way that $\log K_{b}$ exceeds $t^{2} \log K^{a}$, the limit in (7.2) is greater than $2(t+s u) / 3$. By Lemma 5.1, the sequence $\left(\gamma_{j}\right)_{j \geq 1}$ comprises all the best approximants to $\xi$. This implies that we have

$$
w_{2}(\xi)=\lim _{j \rightarrow+\infty} \frac{-\log \left|P\left(\gamma_{j}\right)\right|}{\log H\left(\gamma_{j}\right)}
$$

and

In particular,

$$
w_{2}^{*}(\xi)=\lim _{j \rightarrow+\infty} \frac{-\log \left|\xi-\gamma_{j}\right|}{\log H\left(\gamma_{j}\right)}-1
$$

$$
w_{2}(\xi)-w_{2}^{*}(\xi)=\frac{2(t-1)(t+s u) \log K_{a}+2 s u \log K_{b}}{2(t-1)(t+s u) \log K_{a}+(2 s u+u) \log K_{b}} .
$$


By choosing $a$ and $b$ such that $\sqrt{a}$ and $\sqrt{b}$ do not belong to the same quadratic field and applying the Gelfond-Schneider theorem, this proves that the function $w_{2}-w_{2}^{*}$ takes transcendental values at some automatic continued fractions.

\section{The Thue-Morse continued fraction}

The Thue-Morse sequence $\mathbf{t}=\left(t_{n}\right)_{n \geq 0}$ on the alphabet $\{a, b\}$ is defined as follows: $t_{n}=a$ (respectively, $t_{n}=b$ ) if the sum of binary digits of $n$ is even (respectively, odd). This sequence viewed as the infinite word

$$
\mathbf{t}=a b b a b a a b b a a b a b b a b a a b a b b a a b b a b a a b \ldots
$$

was introduced by Thue [30] in 1912 and then considered nine years later by Morse [24] in a totally different context. It is not ultimately periodic. This is an automatic sequence, also defined as the fixed point beginning with $a$ of the uniform morphism $\tau$ defined by

$$
\tau(a)=a b, \quad \tau(b)=b a .
$$

More information on the Thue-Morse sequence can be found in [9]. Noticing that, for $n \geq 1$, the word $\tau^{n}(a b b a b)=\tau^{n}(a b b) \tau^{n}(a b)$ is a prefix of $\mathbf{t}$, we see that the initial critical exponent of $\mathbf{t}$ is greater than or equal to $5 / 3$.

By a Thue-Morse continued fraction, we mean a real number whose sequence of partial quotients is the Thue-Morse sequence on some alphabet $\{a, b\}$, where $a$ and $b$ are distinct positive integers. Applying a deep result of Schmidt [29] stating that any real algebraic number $\zeta$ of degree at least three satisfies $w_{2}(\zeta)=w_{2}^{*}(\zeta)=2$, M. Queffélec [26] showed in 1998 that the Thue-Morse continued fractions are transcendental. Subsequently [27], she simplified her proof, by showing that any Thue-Morse continued fraction $\xi$ has a Lévy constant (see Theorem 4.3 above). Combined with the fact that ice $(\mathbf{t}) \geq 5 / 3$, this immediately gives, by $(2.3)$,

$$
w_{2}(\xi) \geq w_{2}^{*}(\xi) \geq 7 / 3
$$

and hence, by [29], the transcendence of $\xi$.

An alternative proof was subsequently given in $[2,3]$, based on another result from [29] on the simultaneous approximation of a real number and its square by rational numbers with the same denominator and the fact that arbitrarily long prefixes of $\mathbf{t}$ are palindromes. More precisely, for an irrational real number $\theta$, we let $\lambda_{2}(\theta)$ denote the supremum of the real numbers $\lambda$ for which the inequality

$$
\max \left\{\|q \theta\|,\left\|q \theta^{2}\right\|\right\}<q^{-\lambda}
$$

is satisfied for infinitely many positive integers $q$. Here, $\|\cdot\|$ denotes the distance to the nearest integer. Schmidt [29] established that any real algebraic number $\zeta$ of degree at least three satisfies $\lambda_{2}(\zeta)=1 / 2$. As observed in 
$[2,3]$, if the continued fraction expansion of $\theta$ begins with infinitely many palindromes, then we have

$$
\lambda_{2}(\theta) \geq 1,
$$

and we deduce from Schmidt's result that $\theta$ is either quadratic or transcendental.

This point of view, together with a classical transference principle, allows us to improve the lower bound for $w_{2}(\xi)$ given in (8.1).

Theorem 8.1. Let $\mathbf{a}=a_{1} a_{2} \ldots$ be an infinite word on a finite alphabet composed of positive integers. If arbitrarily long prefixes of a are palindromes and if $\mathbf{a}$ is not ultimately periodic, then the continued fraction

$$
\xi_{\mathbf{a}}:=\left[0 ; a_{1}, a_{2}, \ldots\right]
$$

satisfies $w_{2}\left(\xi_{\mathbf{a}}\right) \geq 3$. Thus, any Thue-Morse continued fraction $\xi$ satisfies $w_{2}(\xi) \geq 3$.

Proof. It suffices to observe that Khintchine's transference principle (see, e.g., Proposition 3.3 in [14]) implies that

$$
w_{2}(\theta) \geq 2 \lambda_{2}(\theta)+1
$$

for any real number $\theta$ not algebraic of degree at most two. Combined with (8.2) this proves the theorem.

We conclude with an open question.

Problem 8.2. Determine $w_{2}(\xi)$ and $w_{2}^{*}(\xi)$ when $\xi$ is a Thue-Morse continued fraction.

The analogue of Problem 8.2 for real numbers whose $b$-ary expansion is a Thue-Morse sequence was solved in [15].

Acknowledgements. The author is very grateful to Jean-Paul Allouche for several useful comments on an earlier version and to the anonymous referee for a very careful reading.

\section{References}

[1] B. Adamczewski And Y. Bugeaud, On the complexity of algebraic numbers, II. Continued fractions, Acta Math. 195 (2005), 1-20.

[2] B. Adamczewski And Y. Bugeaud, A short proof of the transcendence of Thue-Morse continued fractions, Amer. Math. Monthly 114 (2007), 536-540.

[3] B. Adamczewski and Y. Bugeaud, Palindromic continued fractions, Ann. Inst. Fourier (Grenoble) $5 \mathbf{7}$ (2007), 1557-1574.

[4] B. Adamczewski and Y. Bugeaud, Dynamics for $\beta$-shifts and Diophantine approximation, Ergod. Th. Dynam. Syst. 27 (2007), 1695-1711.

[5] B. Adamczewski and Y. Bugeaud, On the complexity of algebraic numbers I. Expansions in integer bases, Ann. of Math. 165 (2007), 547-566. 
[6] B. Adamczewsini et Y. Bugeaud, Mesures de transcendance et aspects quantitatifs de la méthode de Thue-Siegel-Roth-Schmidt, Proc. London Math. Soc. 101 (2010), 1-31.

[7] B. Adamczewski And Y. Bugeaud, Transcendence measures for continued fractions involving repetitive or symmetric patterns, J. Europ. Math. Soc. 12 (2010), 883-914.

[8] B. Adamczewski, Y. Bugeaud et F. Luca, Sur la complexité des nombres algébriques, C. R. Acad. Sci. Paris 339 (2004), 11-14.

[9] J.-P. Allouche And J. Shallit, The ubiquitous Prouhet-Thue-Morse sequence, in Sequences and Their Applications (Singapore, 1998), Springer Ser. Discrete Math. Theor. Comput. Sci., Springer-Verlag, London, (1999), 1-16.

[10] J.-P. Allouche And J. Shallit, Automatic Sequences: Theory, Applications, Generalizations, Cambridge University Press, Cambridge, (2003).

[11] V. Berthé, C. Holton And L. Q. Zamboni, Initial powers of Sturmian sequences, Acta Arith. 122 (2006), 315-347.

[12] Y. Bugeaud, Approximation by algebraic numbers, Cambridge Tracts in Mathematics 160, Cambridge, (2004).

[13] Y. Bugeaud, Diophantine approximation and Cantor sets, Math. Ann. 341 (2008), 677684.

[14] Y. Bugeaud, On simultaneous rational approximation to a real number and its integral powers, Ann. Inst. Fourier (Grenoble) 60 (2010), 2165-2182.

[15] Y. Bugeaud, On the rational approximation to the Thue-Morse-Mahler numbers, Ann. Inst. Fourier (Grenoble) 61 (2011), 2065-2076.

[16] Y. Bugeaud, Variations around a problem of Mahler and Mendès France, J. Aust. Math. Soc. 92 (2012), 37-44.

[17] Y. Bugeaud, Continued fractions with low complexity: Transcendence measures and quadratic approximation, Compos. Math. 148 (2012), 718-750.

[18] Y. Bugeaud, Automatic continued fractions are transcendental or quadratic, Ann. Sci. École Norm. Sup. 46 (2013), 1005-1022.

[19] A. Coвнам, On the Hartmanis-Stearns problem for a class of tag machines, in Conference Record of 1968 Ninth Annual Symposium on Switching and Automata Theory, Schenectady, New York (1968), 51-60.

[20] H. Davenport And W. M. Schmidt, Approximation to real numbers by quadratic irrationals, Acta Arith. 13 (1967), 169-176.

[21] J. F. KoKsma, Über die Mahlersche Klasseneinteilung der transzendenten Zahlen und die Approximation komplexer Zahlen durch algebraische Zahlen, Monats. Math. Phys. 48 (1939), 176-189.

[22] P. LÉvy, Sur le développement en fraction continue d'un nombre choisi au hasard, Compositio Math. 3 (1936), 286-303.

[23] K. MAhLER, Zur Approximation der Exponentialfunktionen und des Logarithmus. I, II, J. reine angew. Math. 166 (1932), 118-150.

[24] M. Morse, Recurrent geodesics on a surface of negative curvature, Trans. Amer. Math. Soc. 22 (1921), 84-100.

[25] O. Perron, Die Lehre von den Ketterbrüchen. Teubner, Leipzig, (1929).

[26] M. QuefFÉLEC, Transcendance des fractions continues de Thue-Morse, J. Number Theory 73 (1998), 201-211.

[27] M. QUEFFÉLEC, Irrational numbers with automaton-generated continued fraction expansion, in Dynamical systems (Luminy-Marseille, 1998), 190-198, World Sci. Publ., River Edge, NJ, (2000).

[28] L. SchaEFfER AND J. Shallit, The critical exponent is computable for automatic sequences, Int. J. Found. Comput. Sci. 23 (2012), 1611-1626. 
[29] W. M. Schmidt, On simultaneous approximations of two algebraic numbers by rationals, Acta Math. 119 (1967), 27-50.

[30] A. ThuE, Über die gegenseitige Lage gleicher Teile gewisser Zeichenreihen, Norske vid. Selsk. Skr. Mat. Nat. Kl. 1 (1912), 1-67. Reprinted in Selected Mathematical Papers of Axel Thue, T. Nagell, ed., Universitetsforlaget, Oslo, (1977), 413-478.

Yann Bugeaud

Université de Strasbourg

Mathématiques

7, rue René Descartes

67084 STRASBOURG (FRANCE)

E-mail: bugeaud@math.unistra.fr 